\title{
Registro De Bienes Culturales Muebles Para El Análisis Arqueológico De Grupos Sociales Precolombinos. Estudio De Caso: Puñay, Cantón Chunchi, Provincia De Chimborazo, Ecuador
}

\author{
Carlos Arturo Jara Santillán \\ Christiam Paúl Aguirre Merino \\ Catalina Margarita Verdugo Bernal \\ Paulina Beatriz Díaz Moyota \\ Sandra Patricia Miranda Salazar \\ Ginno Sidney Jarrín Zambrano
}

Docentes - Investigadores de la Facultad de Recursos Naturales

ESCUELA SUPERIOR POLITÉCNICA DE CHIMBORAZO, ECUADOR

\section{Clara Etelvina Amanta Viveros}

Estudiante, Escuela de Ingeniería en Ecoturismo, Facultad de Recursos

Naturales ESCUELA SUPERIOR POLITÉCNICA DE CHIMBORAZO, ECUADOR

\section{doi: 10.19044/esj.2016.v13n2p440 URL:http://dx.doi.org/10.19044/esj.2016.v13n2p440}

\begin{abstract}
The aim of this study is to present the results of the identification of the cultural styles, social occupations, and cultural affiliates of archaeological sites such as Paccha, Piñancay, Puñay, and Joyaczhi that conforms to the archaeological area, Puñay.

The objective of this study is to keep an archaeological record of the movable cultural property of tourist sites. This is done through the cataloging and characterization of movable property. In cataloging, the records of the archaeological assets of the INPC are applied. Also, in characterization, the methodology was considered as the Crockery for the determination of the Ceramic Cultural styles and the color of the external and internal pulp. In addition, the core of the Munsell tables was considered.

The research allows the identification of 10 ceramic cultural styles in Paccha, 8 in Piñancay, 10 in Puñay sector, and 3 in Joyaczhi through typology and decorative techniques. Also, they are visualized chronologies which are related to the social contexts in which these archeological properties are materialized. The ceramic styles established for the archaeological area
\end{abstract}


belongs to the Integration Period of the pre-Columbian culture Cañari 8001530d.C.

Keywords: Archeology, cultural styles, chronology, Paccha, Piñancay, Puñay and Joyaczhi, pre-Columbian.

\section{Resumen}

El presente artículo se elaboró con la finalidad de presentar los resultados de la identificación de los estilos culturales, ocupaciones sociales y filiaciones culturales de los sitios arqueológicos Paccha, Piñancay, Puñay y Joyaczhi que conforman el área arqueológica Puñay.

El objetivo fue el registrar arqueológicamente los bienes culturales muebles de los sitios mencionados, mediante la catalogación y caracterización de los bienes muebles. Para la catalogación se aplicó las fichas de registro de bienes arqueológicos del INPC. Para la caracterización se consideró la metodología Vajilla para determinar los Estilos Culturales Cerámicos y para la determinación del color de la pasta externa e interna así como también la del núcleo se consideró las tablas Munsell.

La investigación permitió identificar 10 estilos culturales cerámicos en Paccha, 8 en Piñancay, 10 en el sector de Puñay y 3 en Joyaczhi mediante la tipología y técnicas decorativas. Así como también se visualizó cronologías relativas comprendiendo contextos sociales en los cuales se materializaron estos bienes arqueológicos. Los estilos cerámicos establecidos tanto para el área arqueológica pertenecen al Periodo de Integración de la cultura precolombina Cañari 800-1530d.C.

Palabras claves: arqueología, estilos culturales, cronología, Paccha, Piñancay, Puñay y Joyaczhi, precolombina.

\section{INTRODUCCIÓN:}

Según la (Organización de las Naciones Unidas para la Educación, la Ciencia y la Cultura, 2012:3) el patrimonio cultural no se limita a monumentos y colecciones de objetos, sino que comprende también tradiciones o expresiones vivas heredadas de nuestros antepasados $\mathrm{y}$ transmitidas a nuestros descendientes, como tradiciones orales, artes del espectáculo, usos sociales, rituales, actos festivos, conocimientos y prácticas relativos a la naturaleza y el universo, saberes y técnicas vinculados a la artesanía tradicional.

Es importante la conceptualización anterior, para comprender que el patrimonio cultural está definido por aquellos bienes materiales e inmateriales que manifiestan el modo de pensar, sentir y actuar de los pueblos. En cuanto al patrimonio arqueológico, abarca todos los vestigios 
materiales dejados por las sociedades humanas del pasado, como muestra de sus actividades políticas, rituales, económicas, sociales en un tiempo y lugar determinados (Instituto Nacional del Patrimonio Cultural, 2014: 22,23) y que en este artículo se conciben como bienes culturales muebles.

Al hablar de bienes culturales no nos referimos a un inventario de objetos atractivos, factibles de exhibir o yacimientos arqueológicos con arquitectura monumental, sino, al conocimiento e interpretación integral, contextualizado e interrelacionado del patrimonio cultural y su entorno natural, donde los objetos y sitios arqueológicos son solo una parte de un todo, integrados a un sistema cultural que manifiestan procesos históricos únicos e irrepetibles en el tiempo y el espacio. (NAYA, 2012)

Los objetos arqueológicos comprenden los bienes elaborados en diversas materias primas como arcilla, roca, metal, fibras vegetales, conchas, entre otras, y que constituyen la evidencia material del comportamiento humano en las sociedades del pasado. Forman parte de esta categorización los objetos provenientes de investigaciones arqueológicas tanto terrestres como subacuáticas, así como también los bienes que se localizan en museos y colecciones cuya custodia puede ser estatal o particular. (Instituto Nacional del Patrimonio Cultural, 2014: 120)

Actualmente la Escuela Superior Politécnica de Chimborazo se encuentra realizando un proyecto de investigación denominado "Generación de bioconocimiento mediante la recuperación de los saberes ancestrales de la agrobiodiversidad en el área arqueológica del Puñay; el mismo que contempla los sitios de Paccha, Piñancay, Puñay y Joyacshi pertenecientes al Cantón Chunchi.

El cantón Chunchi se encuentra localizado en la provincia de Chimborazo a $124 \mathrm{~km}$ de la ciudad de Riobamba, a $124 \mathrm{~km}$ de la ciudad de Cuenca y a $180 \mathrm{~km}$ de la ciudad de Guayaquil. El clima va desde el subtrópico hasta el frío de los páramos, con una temperatura promedio entre $14^{\circ} \mathrm{C}$. y $21^{\circ} \mathrm{C}$, la humedad relativa es del 60\%, con una pluviosidad de 700 mm anuales promedio. (Gobierno Autónomo Descentralizado Chunchi, 2011: 1)

Según la clasificación ecológica de Chunchi se menciona que el lugar pertenece al Bosque siempreverde montano alto de Cordillera Occidental de los Andes, caracterizada por bosques de 15-20m de altura, con ocasionales árboles emergentes que alcanzan los 30m. (Ministerio Ambiente Ecuatoriano, 2013: 90)

Chunchi está ubicado al centro sur de la provincia de Chimborazo, donde se hallan evidencias arqueológicas del poblamiento de culturas milenarias más tempranas que la cultura Puruhá y que, en el mayor de los casos, estas no se encuentran registradas y documentadas científicamente. Estas evidencias pertenecen al Periodo Formativo (4000a.C. - 300 a.C.) en el 
que se desarrollaron las culturas Narrío y Alausí, su ubicación geográfica se determina en los cantones de Chunchi, Alausí y Guamote; posteriormente en esta misma región en el Periodo de Desarrollo Regional (300 a.C. -500 d.C.) tuvo su apogeo la cultura Cañarí. (Aguirre, 2012: 16).

La Constitución del Ecuador en el Capítulo Cuarto sobre los Derechos de las Comunidades, Pueblos y Nacionalidades, Art. 57., literal 13, manifiesta claramente la necesidad de: "Mantener, recuperar, proteger, desarrollar y preservar su patrimonio cultural e histórico como parte indivisible del patrimonio del Ecuador”. (Constitución Politica del Ecuador, 2008: 9)

Debido a la inexistencia de registros o documentación de la información de estas culturas en el área arqueológica del Puñay, se plantea el registro de bienes culturales muebles para su análisis arqueológico, en virtud de identificar estilos culturales que permitan determinar las ocupaciones de grupos sociales precolombinos y su importancia para el proceso histórico del Ecuador.

\section{METODOLOGÍA}

Para el registro de bienes culturales muebles para el estudio de caso Puñay, que abarca los sitios Paccha, Piñancay, Puñay y Joyacshi, se utilizó:

- Catalogación Cerámica, que documenta de manera científica, sistémica y metodológica los bienes patrimoniales para la elaboración de planes de protección que ayuden en su conservación, preservación, salvaguardia y puesta en valor. (Instituto Nacional del Patrimonio Cultural, 2014: 7).

- Caracterización Cerámica, que determina que en todos los métodos de análisis cerámico existen cuatro variables independientes que tienen que ser consideradas: pasta, tratamiento de superficie, decoración y forma. Todos los ceramólogos deben, en algún punto del análisis, prestar atención a todas estas variables. Sin embargo, puesto que es imposible manejar más de una variable a la vez, la eficacia está en trabajar la forma y técnica decorativa. (Hatch \& Castillo, 1984).

La cerámica utilizada para esta investigación se recolectó del área de influencia del Monte Puñay en cada uno de los cuatro sitios.

- Paccha.- En la comunidad Paccha de la parroquia Llagos, cantón Chunchi, mediante recolección superficial de material cultural.

- Piñancay.- En el área de Piñancay, cantón Chunchi mediante recolección superficial de material cultural.

- Puñay.- Del Bloque Central del Pukará del Puñay. Se realizaron cinco trincheras para obtener material cerámico. 
- Joyacshi.- En la comunidad Joyaczhi de la parroquia Llagos, cantón Chunchi mediante la prospección arqueológica la cual dio como resultados los puntos específicos para proceder a una excavación.

\section{Técnicas e instrumentos de catalogación cerámica}

Se realizó la catalogación del material mediante la ficha de registro de bienes arqueológicos, estipulado en el "Instructivo para fichas de registro e inventario. Bienes arqueológicos” (Instituto Nacional del Patrimonio Cultural, 2014: 119). Ver Anexo A, que considera los siguientes parámetros:

- Datos de identificación

- Datos de localización

- Descripción del bien

- Condición general del bien

- Régimen de custodia

- Condición legal del bien

- Registro del movimiento del bien

- Fotografías adicionales

- Información relacionada

- Bibliografía

- Observaciones

- Datos de control

\section{Técnicas e instrumentos de caracterización cerámica}

Se determinaron los estilos culturales cerámicos, según metodología "Vajilla", la cual, analiza cuatro variables: pasta, tratamiento de superficie, decoración y forma (Hatch, M. 1993: 1-16). Se consideró como información preliminar los estilos cerámicos de Uzcategui (1976), Porras (1977) y Collier y Murra (1942).

Para la determinación del color de la pasta externa e interna, así como también la del núcleo, se consideró las tablas Munsell 2012 que plantea el sistema de ordenación del color de una forma precisa de especificar y mostrar las relaciones entre los colores. Cada color dispone de tres cualidades o atributos: tono, valor y croma o saturación.

Para el análisis estadístico de la caracterización tipológica de la cerámica la información fue sistematizada utilizando el software Microsoft Office Excel 2010. 
RESULTADOS

CATALOGACIÓN CERÁMICA

Sistematización de la cerámica

A partir de la sistematización de la información se estableció: 10 estilos culturales cerámicos en Paccha, 8 estilos culturales cerámicos en Piñancay, 10 estilos culturales en Puñay y 3 estilos culturales cerámicos en Joyagzhi

\section{Cuadro 1. Sistematización de la cerámica}

\begin{tabular}{|c|c|c|}
\hline Sitio & Estilos culturales cerámicos & Número de fragmentos \\
\hline Paccha & $\begin{array}{l}\text { 1. Leonado con ocre pulido (variante A) } \\
\text { 2. Naranja leonado } \\
\text { 3. Leonado } \\
\text { 4. Estilo positivo rojo sobre blanco } \\
\text { 5. Café pulido } \\
\text { 6. Leonado con ocre pulido (variante B) } \\
\text { 7. Naranja con ocre pulido } \\
\text { 8. Ocre pulido } \\
\text { 9. Ocre engobado sobre leonado inciso } \\
\text { 10.Colonial vidriado verdoso }\end{array}$ & 254 \\
\hline Piñancay & $\begin{array}{l}\text { 1. Leonado alisado } \\
\text { 2. Positivo verde blanco } \\
\text { 3. Ocre engobado } \\
\text { 4. Negro sobre ocre } \\
\text { 5. Leonado naranja pulido } \\
\text { 6. Blanco sobre rojo } \\
\text { 7. Rojo sobre leonado grueso } \\
\text { 8. Rojo sobre leonado fino }\end{array}$ & 109 \\
\hline Puñay & $\begin{array}{l}\text { 1. Naranja granulado } \\
\text { 2. Naranja alisado } \\
\text { 3. Leonado engobado - alisado } \\
\text { 4. Gris leonado alisado - grabado } \\
\text { 5. Gris leonado tosco } \\
\text { 6. Rojo pulido sobre leonado } \\
\text { 7. Rojo pulido } \\
\text { 8. Café pulido } \\
\text { 9. Rojo negativo } \\
\text { 10.Rojo engobado sobre leonado inciso }\end{array}$ & 1526 \\
\hline Joyaczhi & $\begin{array}{l}\text { 1. Leonado con ocre pulido (Variante B) } \\
\text { 2. Leonado } \\
\text { 3. Estilo positivo (rojo/blanco) }\end{array}$ & 34 \\
\hline TOTAL & 31 & 1923 \\
\hline
\end{tabular}

Nota: Elaborado por Jara Carlos

Se obtuvo como resultado de la investigación, 31 fichas de registro de bienes arqueológicos, una por cada estilo cultural cerámico identificado en cada uno de los sitios, de un total de 1923 fragmentos. 


\section{CARACTERIZACIÓN CERÁMICA}

\section{Análisis de cerámica del Tambo-Salaceo (Paccha)}

El análisis estadístico de la caracterización tipológica de la cerámica en cuanto a la categoría "Tipo de Fragmento" solamente el 2,36\% pertenecen al tipo "Asa", el 77,17\% a tipo "Borde", el 4,33\% a tipo "Cuello-labio", y el 3,15\% a tipo "Cuerpo". Mientras que el 12,99\% son fragmentos "Sin identificación”. De un total de 254 fragmentos analizados.

Figura 1. Tipos de fragmentos cerámicos del Tambo Salaceo (Paccha)

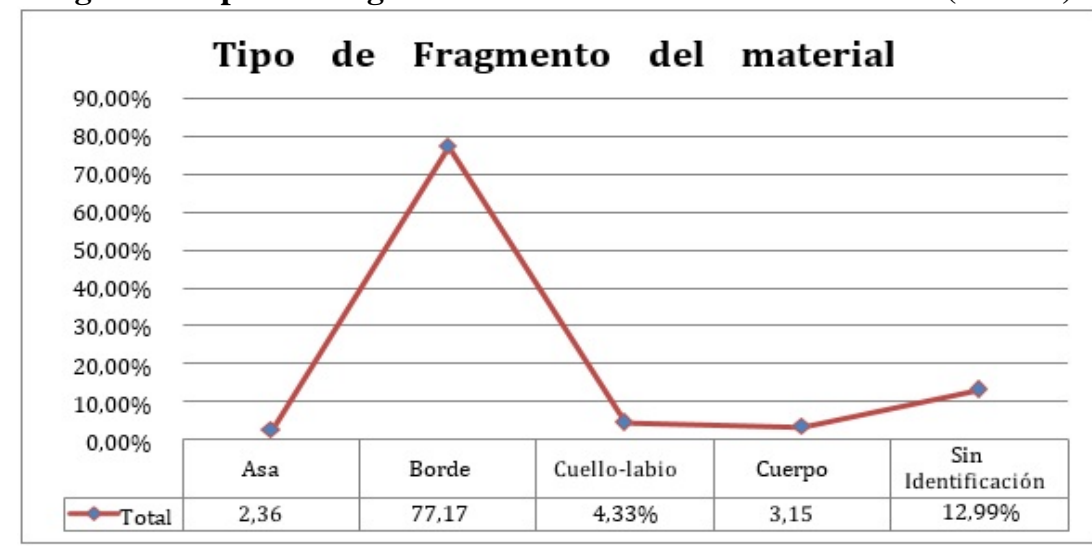

Nota: En (Amanta, Clara, pág.126)

En cuanto a los estilos culturales identificados se encuentra como predominante el leonado con ocre pulido (Variante A) con el 30,31\%, seguido de los estilos naranja con ocre pulido con el 22,05\%, leonado con el $17,32 \%$, ocre engobado sobre leonado inciso con el 9,45\%, leonado con ocre pulido (Variante B) con el 7,09\%, naranja leonado con el 7,09\%, positivo rojo sobre blanco con el 2,76\%, ocre pulido con el 1,97\%, café pulido con el $1,57 \%$ y colonial vidriado verdoso con el $0,39 \%$.

Figura 2. Estilos culturales cerámicos del Tambo Salaceo (Paccha)

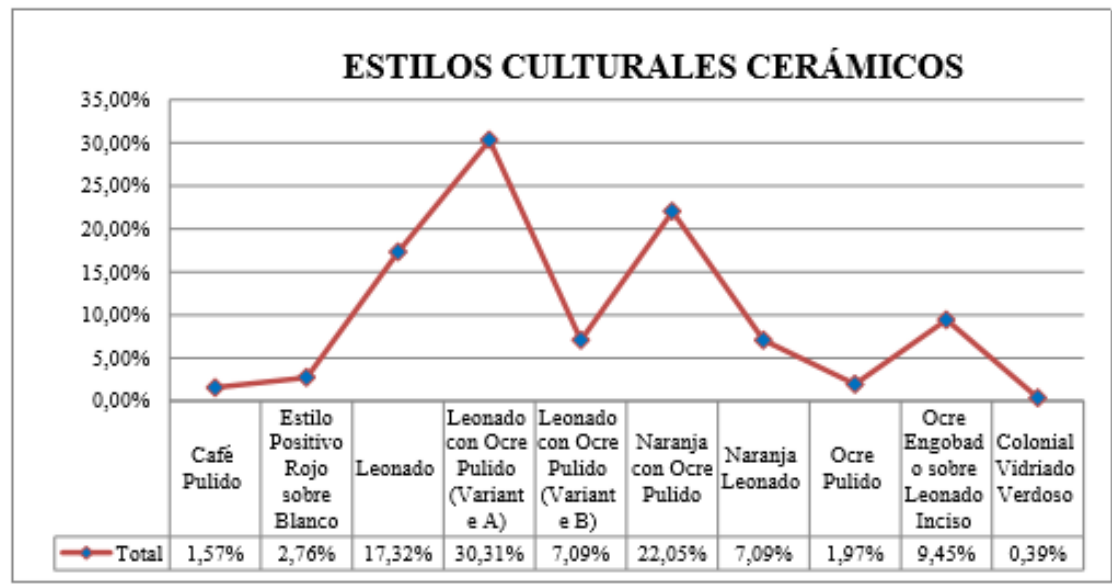

Nota: En (Amanta, Clara, pág.127) 
Análisis de cerámica de Piñancay

El análisis estadístico de la caracterización tipológica de la cerámica en cuanto a la categoría "Tipo de Fragmento" solamente el 0,93\% pertenecen a tipo "Base”, el 44,95\% a tipo "Borde”, el 2,75\% a tipo "Cuello", el 2,75\% a tipo "Cuello-labio”, y el 13,76\% a tipo “Cuerpo". Mientras que el 34,86\% son fragmentos “Sin identificación”. De un total de 109 fragmentos analizados que fueron tomados porque presentaban rasgos decorativos para poder determinar los estilos culturales.

Figura 3. Tipos de fragmentos cerámicos de Piñancay

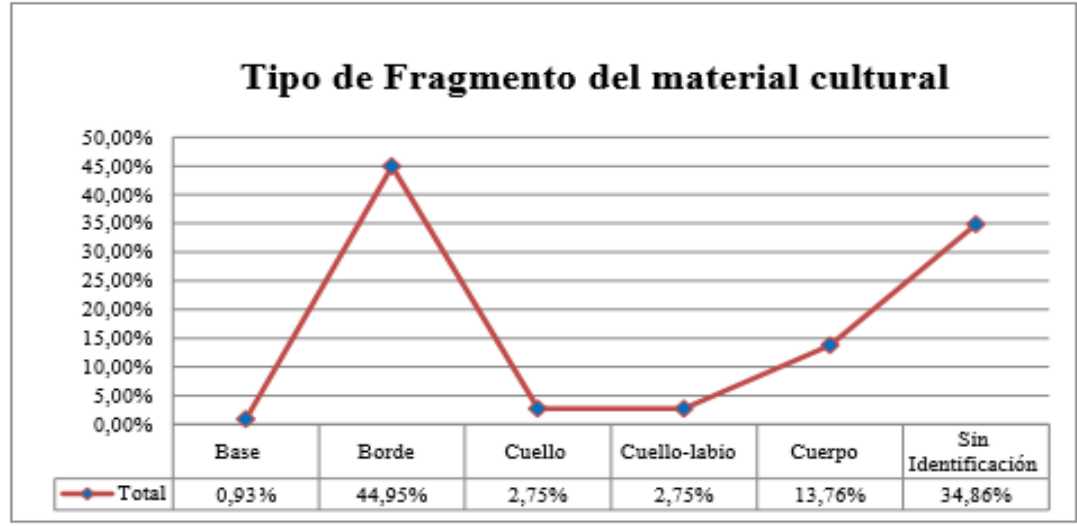

Nota: En (Amanta, Clara, pág.138)

En cuanto a los estilos culturales identificados se encuentra como predominante el leonado naranja pulido con el 27,52\%, seguido de los estilos rojo sobre leonado grueso con el 22,02\%, rojo sobre leonado fino con el $12,85 \%$, ocre engobado con el $11,92 \%$, blanco sobre rojo con el $10,09 \%$, negro sobre ocre con el 8,26\%, positivo verde blanco con el 4,59\%, y leonado alisado con el 2,75\%.

Figura 4. Estilos culturales cerámicos de Piñancay

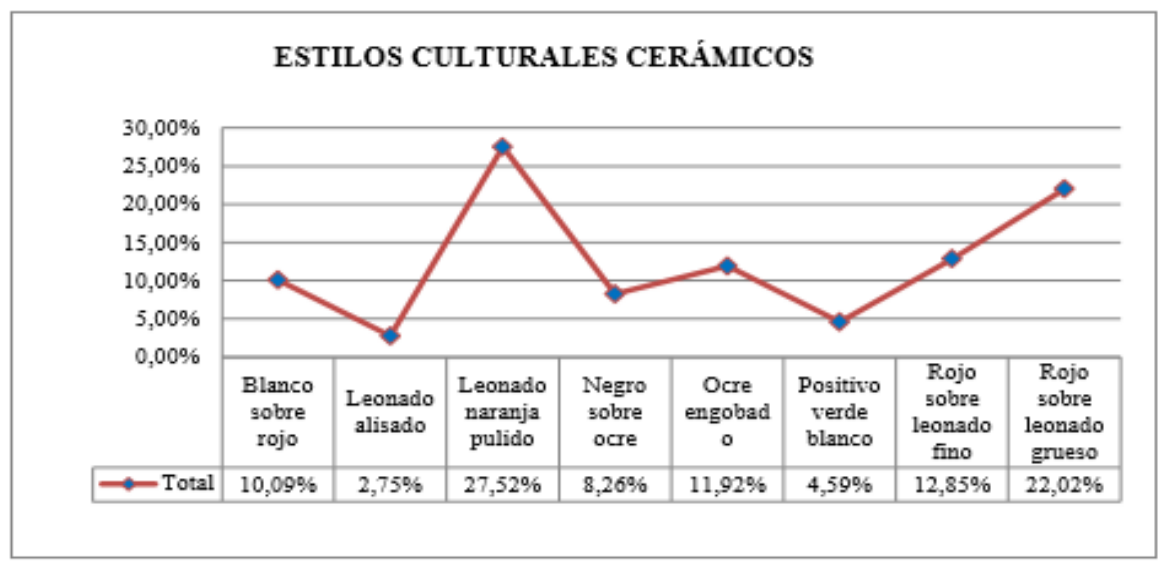

Nota: En (Amanta, Clara, pág.139) 
Análisis de cerámica del Puñay

De un total de 1526 fragmentos, el 69.13\% pertenecen a la Trinchera 03, el $14.98 \%$ a la Trinchera 01, el $12.95 \%$ a la Trinchera 05 y el $2.94 \%$ a la Trinchera 02. Cabe señalar que en la Trinchera 04 no se encontró ningún fragmento de cerámica o material cultural alguno.

Figura 5. Fragmentos cerámicos hallados en las Trincheras

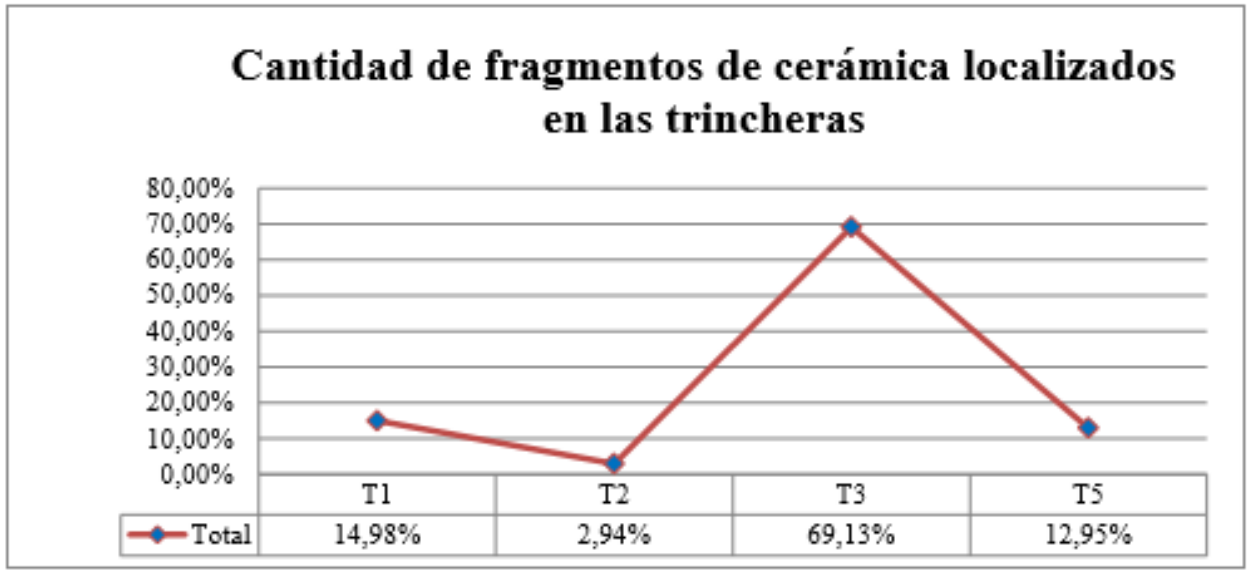

Nota: En (Amanta, Clara, pág.148)

En cuanto a la categoría del "Tipo de Fragmento", solamente el 0.91\% pertenecen al tipo “Base”, el 4.84\% pertenecen al tipo “Borde”, el 0.13\% a tipo “Cuello” y el 0.07\% a tipo “Cuerpo”. Mientras que el 94,05\% son fragmentos “Sin identificación”.

Figura 6. Tipos de fragmentos cerámicos del Puñay

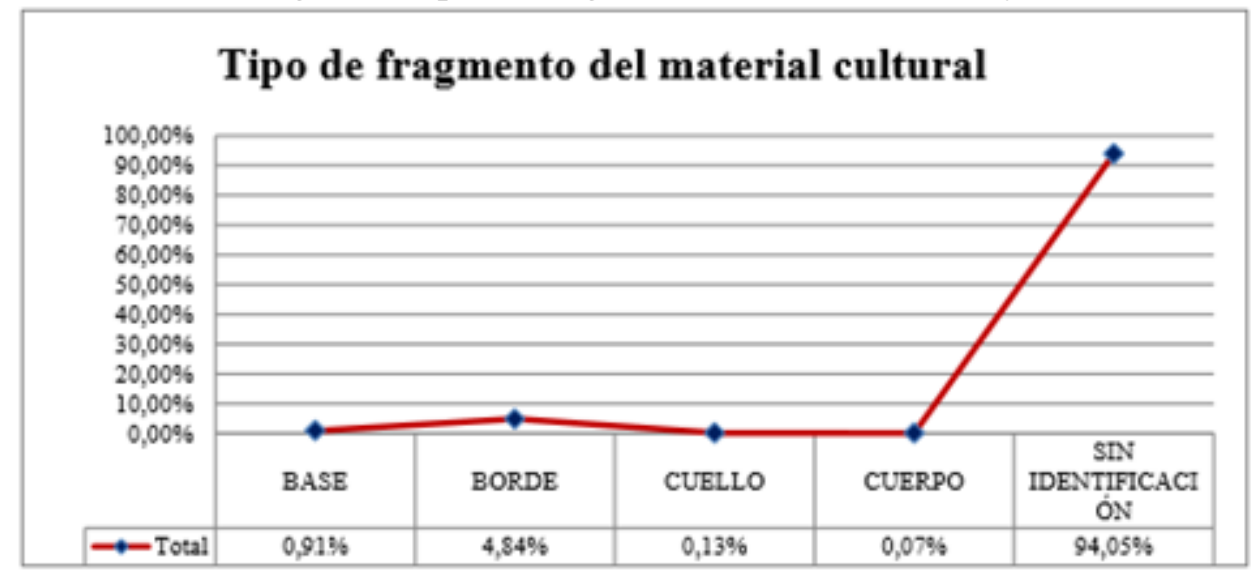

Nota: En (Amanta, Clara, pág.148)

Como se puede apreciar el estilo de pasta predominante es el gris leonado alisado - grabado con el 43.75\%, seguido de los estilos leonado engobado - alisado con el 17.46\%, naranja granulado con el 13.15\%, 
café pulido con el 7.30\%; gris leonado tosco con el 5.89\%, rojo engobado sobre leonado inciso con el 5.69\%, naranja alisado con el $4.32 \%$, rojo pulido con el 1.31\%, rojo pulido sobre leonado con el $1.05 \%$ y rojo negativo con el $0.07 \%$.

Figura 7. Estilos culturales cerámicos del Puñay

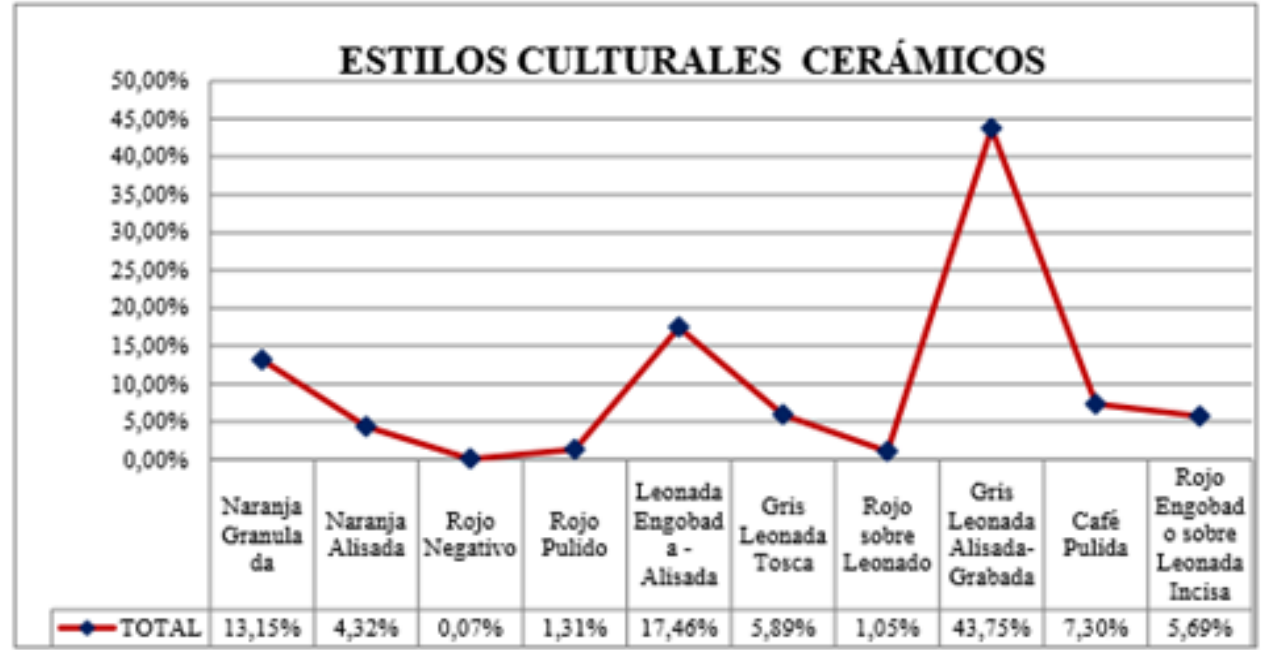

Nota: En (Amanta, Clara, pág.149)

Análisis de cerámica de Joyaczhi

De 34 fragmentos analizados en dos trincheras efectuadas en las terrazas arqueológicas de Joyaczhi, el 61,11\% pertenecen a la Trinchera 01 y el 38,89\% a la Trinchera 02.

Figura 8. Fragmentos cerámicos hallados en las Trincheras

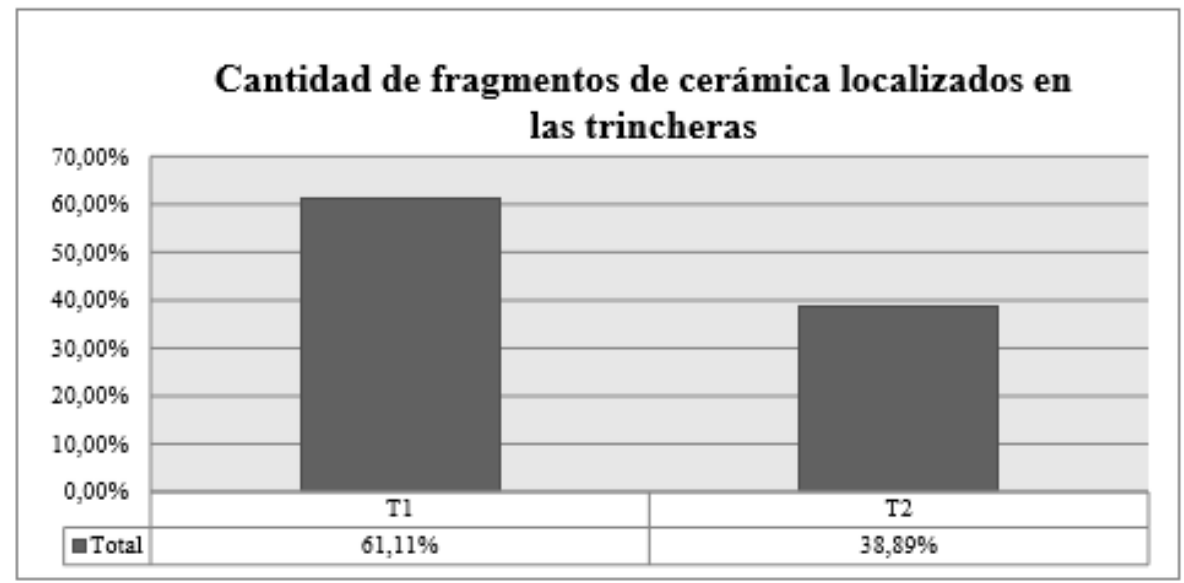

Nota: En (Amanta, Clara, pág.149)

El análisis estadístico de la caracterización tipológica de la cerámica en cuanto a la categoría “Tipo de Fragmento” solamente el 5,56\% 
pertenecen a tipo “Asa”, el 5,56\% a tipo "Base”, el 5,56\% a tipo "Cuello”, el 16,67\% a tipo "Cuello-labio”, el 27,78\% a tipo “Cuerpo” y el 38,87\% a tipo "Borde".

Figura 9. Tipos de fragmentos cerámicos de Joyaczhi

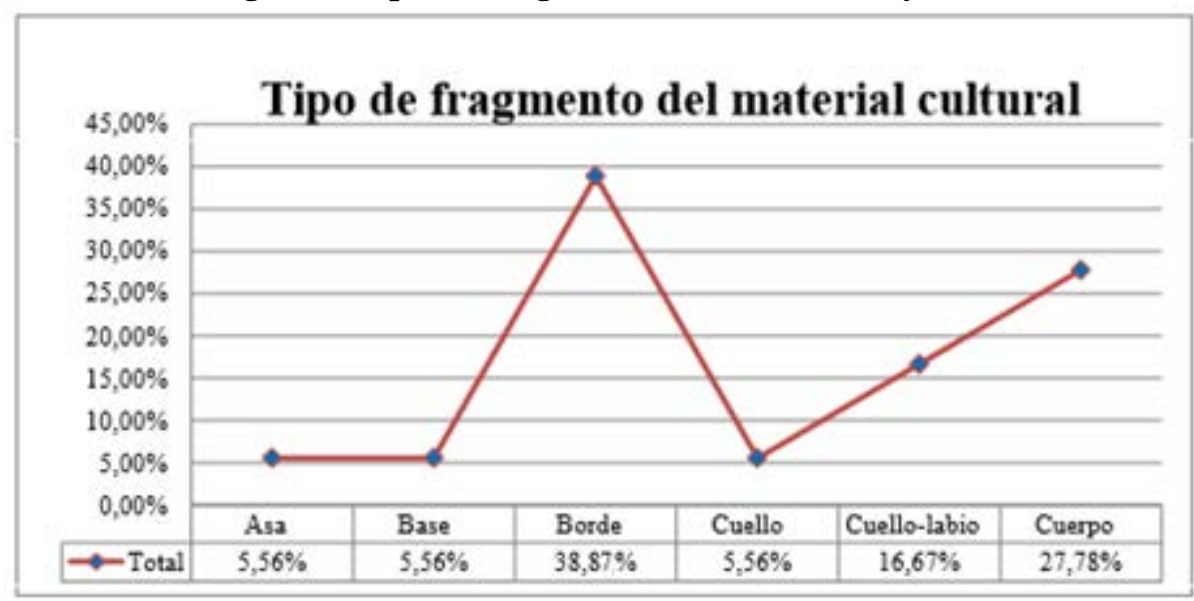

Nota: En (Amanta, Clara, pág.149)

En cuanto a los estilos culturales identificados se encuentra como predominante el leonado con ocre pulido (Variante B) con el 61,11\%, seguido de los estilos leonado con el 33,33\%, y positivo rojo sobre blanco con el 5,56\%.

Figura 10. Estilos culturales cerámicos de Joyaczhi

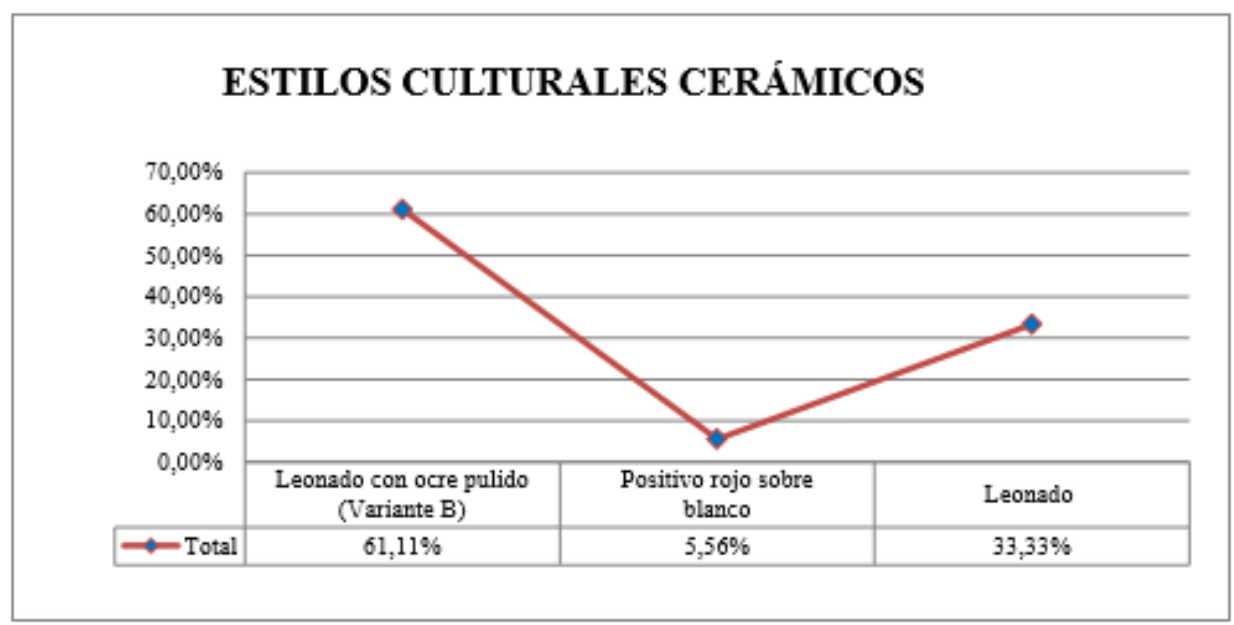

Nota: En (Amanta, Clara, pág.149)

\section{CONCLUSION}

- A partir de la catalogación de cerámica se estableció diez estilos culturales cerámicos en el sector de Paccha los cuales son: leonado con 
ocre pulido (Variante A), naranja con ocre pulido, leonado, ocre engobado sobre leonado inciso, leonado con ocre pulido (Variante B), naranja leonado, positivo rojo sobre blanco, ocre pulido, café pulido y colonial vidriado verdoso.

- Se estableció ocho estilos culturales cerámicos en el sector de Piñancay los cuales son: leonado naranja, rojo sobre leonado grueso, rojo sobre leonado fino, ocre engobado, blanco sobre rojo, negro sobre ocre, positivo verde blanco y leonado alisado.

- También se estableció diez estilos culturales cerámicos en el sector de Puñay los cuales son: gris leonado alisado - grabado, leonado engobado - alisado, naranja granulado, café pulido, gris leonado tosco, rojo engobado sobre leonado inciso, naranja alisado, rojo pulido, rojo pulido sobre leonado y rojo negativo.

- Además se estableció tres estilos culturales cerámicos en el sector de Joyaczhi los cuales son: leonado con ocre pulido (Variante B), leonado y positivo rojo sobre blanco.

- El estilo cultural cerámico leonado con ocre pulido (Variante B) está presente en Paccha y Joyaczhi, el estilo cultural cerámico leonado está presente en Paccha, Puñay y Joyaczhi, el estilo cultural cerámico positivo rojo sobre blanco también está presente en Paccha y Joyaczhi, estos resultados nos muestran que en efecto hubo una filiación cultural en un mismo periodo y que aquellas poblaciones estaban conectadas entre sí.

- Los estilos cerámicos establecidos tanto para el área arqueológica de Paccha, Piñancay, Puñay y Joyaczhi pertenecen al periodo de Integración de la cultura Cañari 800-1530d.C. puesto que los fragmentos de cerámica muestran que las técnicas de elaboración así como el diseño y tratamiento son propias de esta cultura, en un objeto icónico como es la olla con incisiones lineales en forma de triángulo que bordean otras incisiones circulares, cuello estrecho, con asas redondeadas y grandes.

- En el área de investigación de Paccha se encontró además un Estilo cultural Colonial vidriado verdoso que pertenece al periodo Colonial 1530-1830d.C. el cual presenta un acabado de superficie vidriado y presenta líneas entrecruzadas de color verde sobre la pasta interna.

- En cuanto a la caracterización cerámica en el área de Paccha se encontró la mayoría de fragmentos de tipo borde y el estilo cultural predominante es el leonado con ocre pulido (Variante B), en el área de Piñancay la mayoría de fragmentos son de tipo borde y el estilo cultural predominante es el Leonado naranja pulido, en el área del Puñay la mayoría de fragmentos son de tipo borde y el estilo cultural 
predominante es gris leonado alisado-grabada y en el área de Joyaczhi la mayoría de fragmentos son de tipo borde y el estilo cultural predominante es el leonado con ocre pulido (Variante B).

\section{References:}

1. Organización de las Naciones Unidas para la Educación, la Ciencia y la Cultura. (2012). Qué es el patrimonio cultural inmaterial? pp. 3.

2. Instituto Nacional del Patrimonio Cultural. (2014). Instructivo para fichas de registro e inventario Bienes Arqueológicos. pp. 7, 22, 23, 27, 119, 120.

3. NAYA. (2012). Patrimonio Arqueológico. Recuperado el 15 de Abril del 2016 de: http://www.equiponaya.com.ar/turismo/postura_turismo_y_patrimo nio.htm

4. Gobierno Autónomo Descentralizado Chunchi. (2011). Plan de Desarrollo y Ordenamiento Territorial Chunchi. pp. 1.

5. Ministerio Ambiente Ecuatoriano. (2013). Sistema de clasificación de los ecosistemas de Ecuador Continental. pp. 90.

6. Aguirre, C. (2012). Ruta arqueológica de los andes. Informe Puñay INPC. pp. 16.

7. Constitución Política del Ecuador. (2008). Derecho de las comunidades, pueblos y nacionalidades. pp. 9.

8. Hatch, M., \& Castillo, D. (1984). Método simplificado para la clasificación de cerámica arqueológica. pp. 80-91.

9. Munsell, A. H. (2012). Sistema Munsell de especificación de color. Recuperado el 18 de Abril del 2016 de: http://redgeomatica.rediris.es/carto2/arbolB/cartoB/Bcap5/5_9_1.ht $\mathrm{m}$

10. Amanta Viveros, C. E. (2016). Registro arqueológico de los bienes culturales muebles de los sitios: Paccha, Piñancay, Puñay y Joyaczhi; cantón Chunchi, provincia de Chimborazo, Ecuador 
Anexo A. Ficha de registro de bienes arqueológicos

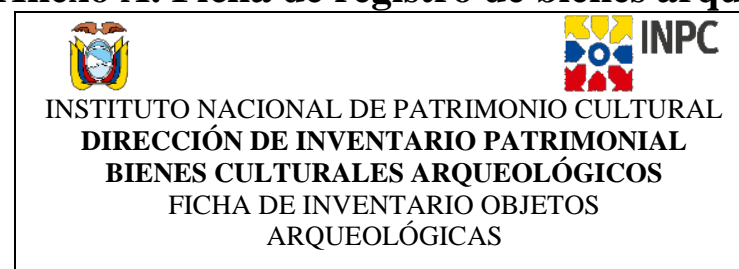

\begin{tabular}{|l|}
\hline \\
\hline$N^{\circ}$ de \\
\hline Código de colección \\
\hline
\end{tabular}

\section{DATOS DE IDENTIFICACIÓN}

\begin{tabular}{|c|c|c|}
\hline Bien cultural: & Material: & \multirow{2}{*}{ Fotografía principal } \\
\hline Periodo histórico: & Cronología: & \\
\hline Filiación cultural: & Descripción de la fotografía: \\
\hline \multicolumn{2}{|c|}{ 62.DATOS DE LOCALIZACIÓN } \\
\hline \multicolumn{2}{|c|}{}
\end{tabular}

\begin{tabular}{|c|c|c|c|c|}
\hline Provincia: & Cantón: & \multicolumn{2}{|c|}{ Parroquia: } & Recinto/Comunidad \\
\hline & & Urbana: & Rural: & \\
\hline \multicolumn{3}{|c|}{ Contenedor inmueble: } & \multicolumn{2}{|c|}{ Subcontenedor: } \\
\hline
\end{tabular}

\section{Dirección: \\ Colección:}

Coordenadas:

$\begin{array}{lcc}\text { Este(X): } & \text { Norte(Y): } & \text { Altitud(Z): }\end{array}$

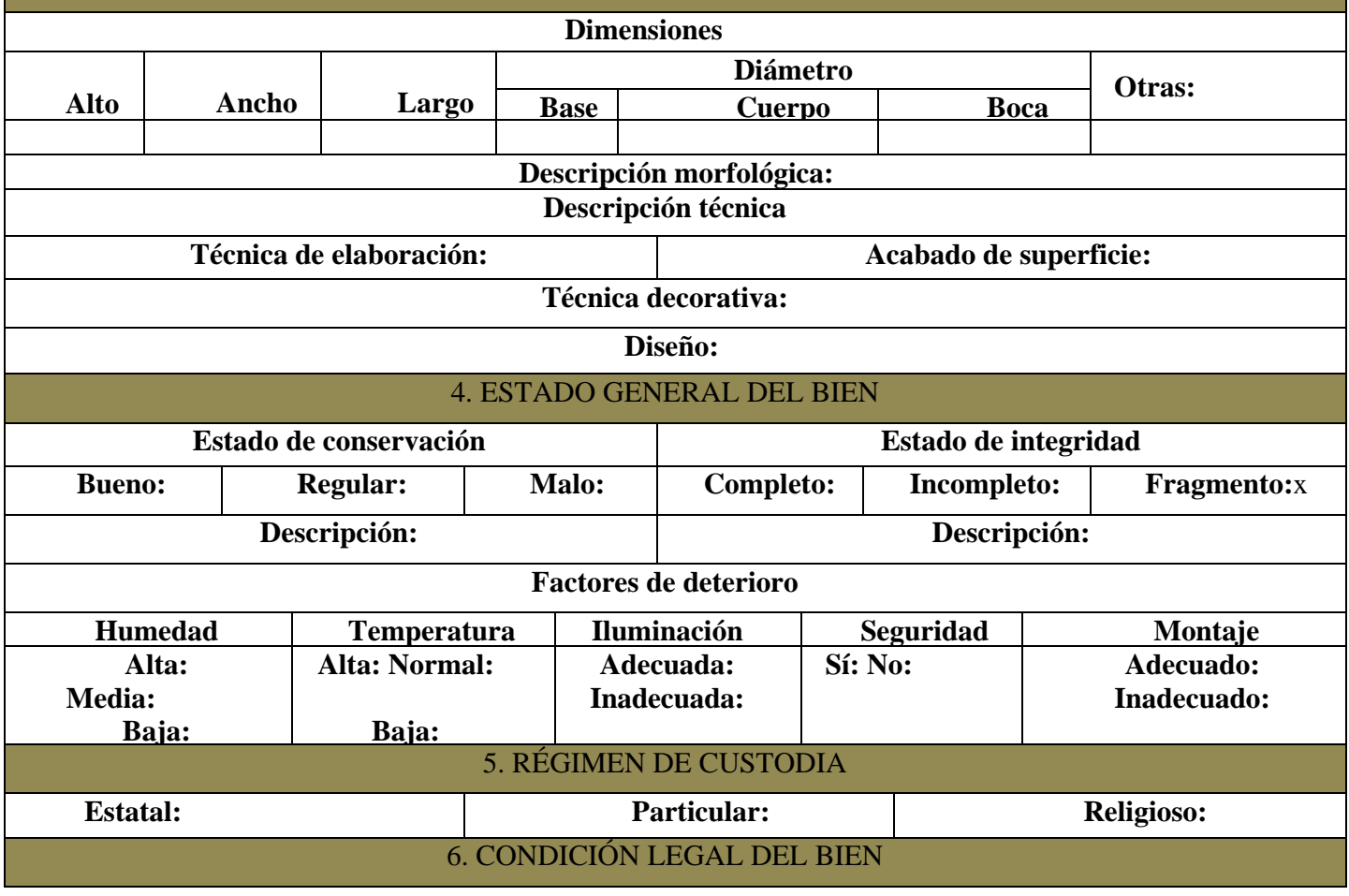




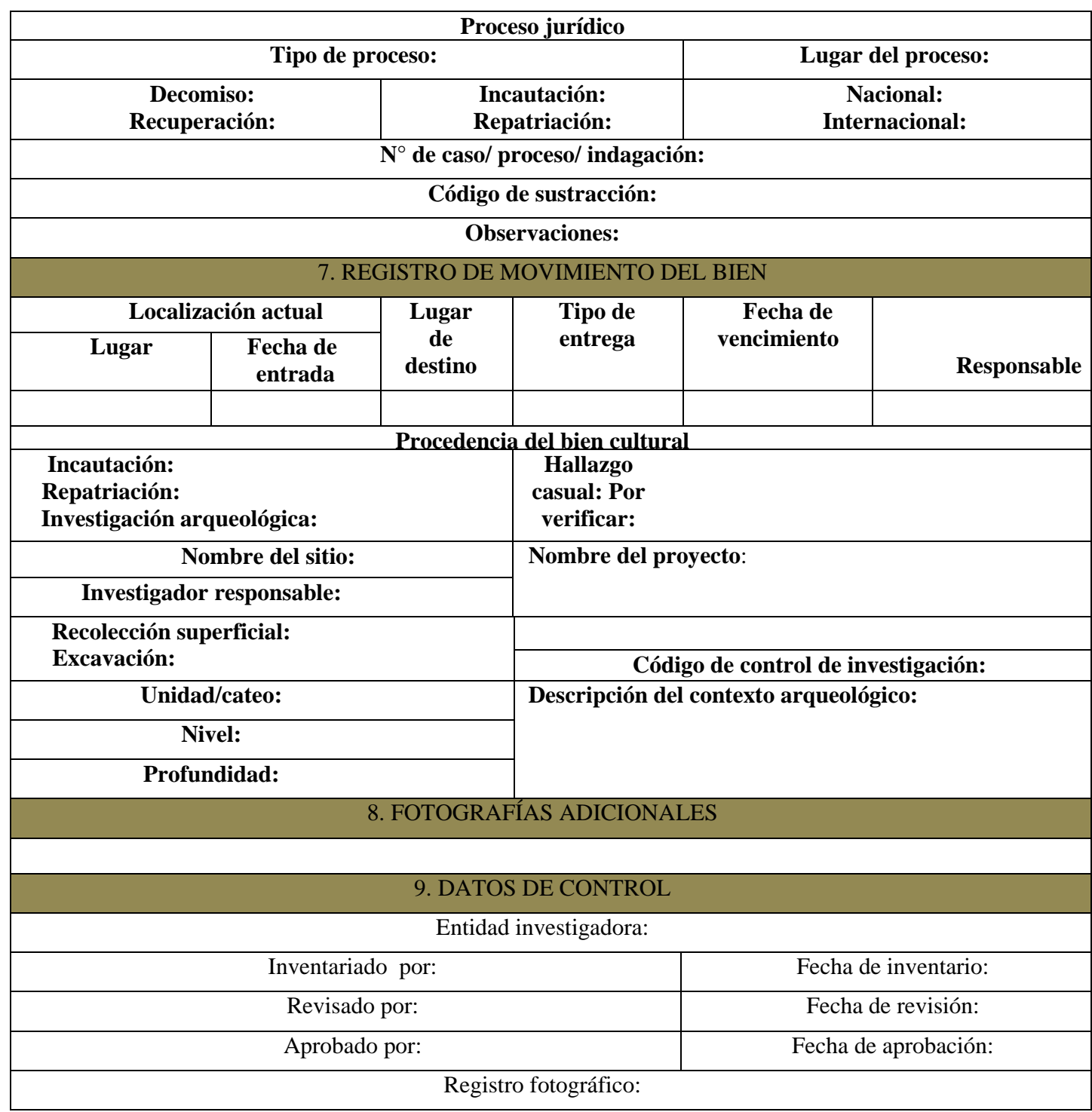

Nota: En (Instructivo para fichas de registro e inventario Bienes Arqueológicos, 2014, pág.

27) 